\title{
Is Xysticus kochi (Araneae: Thomisidae) an efficient indigenous biocontrol agent of Frankliniella occidentalis (Thysanoptera: Thripidae)?
}

\author{
Peter Zrubecz $\cdot$ Ferenc Toth $\cdot$ Attila Nagy
}

Published online: 1 April 2008

(C) International Organization for Biological Control (IOBC) 2008

\section{Erratum to: BioControl \\ DOI 10.1007/s10526-007-9100-6}

Incorrect SD values were published in Table 1 of the original article. The correct values are shown in Table 1 below. Since the mistake did not affect the statistical analysis, the authors do not modify the statements written in the original paper.

Table 1 Effects of introducing $X$. kochi spiderlings and supplementing of mulch to caged sweet pepper plants infested with thrips on mean $( \pm \mathrm{SD}$, in $\mathrm{g})$ weight of fruits classified according to marketability (rows)

\begin{tabular}{llllll}
\hline & Control & Infested & Mulch & Xysticus & Xysticus + Mulch \\
\hline Fresh market & $322.63 \pm 320.78 \mathrm{a}$ & $87.19 \pm 87.14 \mathrm{a}$ & $102.70 \pm 121.61 \mathrm{a}$ & $753.46 \pm 194.51 \mathrm{~b}$ & $809.95 \pm 379.99 \mathrm{~b}$ \\
Canning & $194.45 \pm 100.54 \mathrm{a}$ & $127.65 \pm 149.68 \mathrm{a}$ & $211.52 \pm 166.51 \mathrm{a}$ & $211.43 \pm 131.66 \mathrm{a}$ & $87.71 \pm 96.14 \mathrm{a}$ \\
Sliced & $244.22 \pm 180.66 \mathrm{a}$ & $192.45 \pm 214.34 \mathrm{ab}$ & $354.08 \pm 261.14 \mathrm{a}$ & $49.01 \pm 87.45 \mathrm{~b}$ & $43.45 \pm 94.71 \mathrm{~b}$ \\
$\begin{array}{l}\text { Non- } \\
\quad \text { marketable }\end{array}$ & 0.00 & $175.54 \pm 206.84 \mathrm{a}$ & $58.04 \pm 122.70 \mathrm{a}$ & $10.26 *$ & 0.00 \\
\hline
\end{tabular}

Aesthetical damage was caused by Frankliniella occidentalis and Frankliniella intonsa, except in the 'Control' treatment where it was exclusively from $F$. intonsa. Means within a row followed by the same letter are not significantly different (Tukey's HSD test, $p>0.05$ ). $\mathrm{N}=70$

* only a single non-marketable pepper was harvested

The online version of the original article can be found under doi:10.1007/s10526-007-9100-6.

P. Zrubecz $(\bowtie) \cdot$ F. Toth · A. Nagy

Szent Istvan University, Faculty of Agricultural and Environmental Sciences, Department Plant Protection, Pater K. u. 1, Godollo 2103, Hungary

e-mail: pzrubecz@gmail.com

F. Toth

e-mail: toth.ferenc@mkk.szie.hu

A. Nagy

e-mail: attila.nagy@mkk.szie.hu 